\title{
Marco para una discusión sobre educación: la ciencia y la tecnología, aspectos claves de la soberanía nacional
}

\author{
Edgar Augusto Andrade Londoño \\ Decano de la Facultad de Ciencia y Tecnología \\ Universidad Pedagógica Nacional \\ M.Sc., U. de California-Berkeley \\ B. Sc., Lafayette College.
}

\begin{abstract}
RESUMEN
Se presentan aquí consideraciones acerca de las realidades mundiales y nacionales que sustentan la opinión de que la educación es el medio más sistemático y estable para la difusión de la Ciencia y la Tecnología. El desarrollo autónomo de Colombia es imposible mientras no se transciendan los límites de la División Internacional del Trabajo impuesta por las grandes potencias, y no se consolide un mercado interno que sustente el progreso nacional. El país debe definir prioridades para su desarrollo científicotecnológico, acentuando primeramente la investigación tecnológica.

En medio de las múltiples discusiones en curso sobre los fines y propósitos, sobre la axiología, los contenidos y los métodos de la educación, se han venido abriendo paso dos consensos importantes.

El primero de ellos, reside en que las propuestas educativas deben tener como referencia el marco más amplio del panorama nacional, en particular, una definición sobre la contribución que el sistema educativo puede hacer para la superación de los grandes problemas colombianos. El segundo, que de alguna manera la ciencia y la tecnología desempeñan un papel decisivo para nuestro desarrollo como país $\mathrm{y}$, por lo tanto, también deben jugar un rol central en los esfuerzos educativos.

En diversas oportunidades hemos sostenido (1) que la educación debe ser el medio más estable y sistemático para la difusión de las ciencias y la tecnología en una sociedad contemporánea, y que, en esa calidad, la educación no es una condición suficiente pero sí necesaria para nuestro desarrollo nacional autónomo. Es mirando la educación dentro de esta perspectiva, que podremos efectuar y experimentar propuestas relevantes y viables en nuestras actuales condiciones sociales. El propósito del presente artículo es presentar, de manera algo más detallada, la concepción de las realidades globales y nacionales que nutren dicha opinión con la esperanza de colaborar al enriquecimiento del debate.
\end{abstract}




\section{EL PROGRESO Y LA SOBERANIA NACIONALES \\ REQUIEREN COMO BASE MATERIAL DE UN DESARROLLO ECONOMICO SUSTENTADO EN LA APLICACIÓN DE LA CIENCIA Y LA TECNOLOGIA AL APARATO PRODUCTIVO COLOMBIANO.}

\subsection{La Política de la División Internacional del Trabajo.}

Desde finales de la década de los sesentas, tanto los Estados Unidos como la Unión Soviética, definieron explícitamente la División Internacional del Trabajo como principio rector de sus relaciones económicas con sus respectivas zonas de influencia. Aplicada como la política del "buen socio" desde la presidencia de R. Nixon en el caso de Occidente, y a través de las decisiones del CAME en el caso de Europa Oriental y Cuba, consiste esencialmente en que la potencia central se reserva para sí el desarrollo de la industria intensiva en capital, esto es, de alta tecnología; al mismo tiempo asigna al resto de países de su esfera el papel de proveedores de materias primas, mano de obra barata y de productos industriales intensivos en mano de obra. "De esta manera" según lo explicaba N. Rockefeller, a la sazón Vicepresidente Norteamericano" el beneficio (del capital) será mayor" (2).

En la implementación de esta desigual estrategia de asociación, el problema de los socios en los países periféricos estaba resuelto para la Unión Soviética. Todos esos países cuentan con una economía altamente centralizada y dirigida por el Estado. Paradójicamente, para los Estados Unidos no existían inversionistas privados en sus países periféricos con la capacidad necesaria y recurrieron también al Estado como socio principal.

Complementada con el hecho de que, desde los años cincuentas, la exportación de capital desde los Estados Unidos se hace prioritariamente por medio de préstamos y no de inversión directa o donaciones, en dos décadas la División Internacional del Trabajo produjo los siguientes resultados:

- Una ampliación de la brecha tecnológica que separa a las potencias centrales de los países periféricos.

- El deterioro de los términos de intercambio entre la potencia y su periferia. Los productos industriales intensivos en capital importados por la periferia son cada vez más costosos para éstos mientras que sus bienes terminados, productos agrícolas o recursos naturales son más baratos en los mercados internacionales.

El necesario crecimiento del endeudamiento externo de los países de la periferia.

- Un desmesurado crecimiento del sector estatizado de la economía en los países de América Latina, dirigido por un Estado burocratizado, ineficiente y corrompido.

- La consolidación de las actividades especulativas y financieras por encima de las actividades productivas estancadas en los países periféricos por falta de rentabilidad, apoyo oficial, y por la competencia de la industria de las potencias.

- La consecuente crisis económica que agobia a los países periféricos. 


\subsection{Las consecuencias de la División Internacional del Trabajo en Colombia.}

En Colombia la planificación estatal de la economía data de los primeros años de la década de los cincuentas, como resultado no del desarrollo interno sino de la aplicación del pacto de Breton Woods y la conformación del nuevo sistema financiero internacional bajo la égida de los Estados Unidos a través del Fondo Monetario Internacional. Esa experiencia facilitó la reforma constitucional de 1968 que acondicionó al aparato estatal colombiano a las necesidades de la aplicación de la política de asociación; la creación del sistema UPAC en 1972, que afianzó al sector financiero de la economía y, finalmente, la clara explicitación en 1976 de la forma asociativa con el capital extranjero para la explotación de nuestros recursos naturales.

Así la planeación económica por parte del Estado, a cargo de distintos gobiernos desde 1968, se ha efectuado sobre la base de dos axiomas centrales: primero, que el desarrollo nacional no es posible sin los empréstitos y la inversión extranjeros y en segundo término el acondicionamiento ala política de la División Internacional del Trabajo. Las consecuencias pueden verse en los siguientes aspectos:

Ahondamiento de la Brecha Tecnológica.

Algunos indicadores(3) para comparar los esfuerzos en ciencia y tecnología de los países son:

Científicos (1988): $\quad \%$ total mundial

Unión Soviética

Europa

Estados Unidos - Canadá

América Latina
36

22.4

18.5

2.4

Colombia aporta el $4.5 \%$ del porcentaje de América Latina.

\section{Recursos Financieros para la investigación en Ciencia y Tecnología (1988) por países:}

Europa

Estados Unidos - Canadá

América Latina
$\%$ total mundial

34

32

1.8

Colombia aporta el $1.4 \%$ del porcentaje de América Latina. 


\section{Inversión en C.T. por habitante (1988)}

Estados Unidos

Brasil

Colombia
500 dolares

22 dolares

1 dolar

Crecimiento de la productividad (1950-1975):

Japón

Alemania
Estados Unidos

Colombia

\section{$239 \%$}

141

134

91

El deterioro de los términos de intercambio:

La sostenida devaluación del peso colombiano frente al dólar de los Estados Unidos ha incidido tan evidentemente en el encarecimiento de nuestras importaciones, que no se necesita de mayor sustentación. Por el otro lado, basta señalar lo siguiente sobre nuestros principales recursos naturales explotados dentro de la política de asociación.

- Colombia comenzó a exportar petróleo y ferroníquel en momentos en que el precio internacional estaba deprimido.

- Los costos de producción de la libra de ferroníquel (Cerromatoso) y de la tonelada de carbón (Cerrejón) superan los precios de venta. En este último caso, para 1987 el costo de producción era de US\$33/ton. mientras que el precio de venta promediaba los US\$ 28/ton.

\section{El endeudamiento Externo:}

En términos absolutos, la deuda externa colombiana creció de unos US\$5 mil millones a comienzos de los años ochentas, a unos US\$ I8mil millones en la actualidad. Más preocupante aún es que desde 1978 el pago de intereses superó las amortizaciones de capital, y el servicio de la deuda demanda al año una cantidad equivalente alas exportaciones de café (unos US\$1 mil millones).

\section{El crecimiento del Sector Estatal de la Economía:}

Es bien conocido el hecho de que el Estado es el mayor empleador en Colombia (cerca del $12 \%$ de la población económicamente activa, mientras que la industria sólo emplea el 4\%). La participación del sector público en el Producto Interno Bruto (PIB) pasó del $14 \%$ en 1970 al $18 \%$ en 1980.

Igualmente conocida es la práctica de que las pérdidas (y/o desfalcos) de las grandes empresas colombianas son patrimonio público, pero las ganancias son privadas. Así ha 
ocurrido con la banca (la creación del Fondo de Garantías después de los escándalos de 1985), con las inversiones industriales del IFI, y por supuesto con los contratos de asociación. En este último aspecto, numerosos estudiosos (4) de los casos de Cerrejón y Cerromatoso han coincidido en señalar cómo a través de una compleja red de subsidiarias; de la práctica de la sobre y sub-facturación y de los desiguales términos de contratación, las grandes multinacionales obtienen réditos de su inversión mientras que su socio, el estado colombiano, tiene que absorber las consecuencias de la diferencia entre los precios de producción y venta.

\section{La Consolidación del Sector Financiero a costa de los Sectores Productivos:}

Las siguientes estadísticas que muestran las variaciones en la participación en el PIB, ilustran el caso:

\section{Participación en el PIB (\%) 195019701980}

Agricultura

Industria

Financiero más alquiler vivienda

\section{$38 \quad 25 \quad 22$}

$14 \quad 20 \quad 14$

810

Desde la implantación del sistema UPAC en 1972, la inversión especulativa pasó a ser más rentable que la inversión productiva. Esto condujo, además, a la conformación de una mentalidad de rápido y fácil enriquecimiento entre nuestra población que sirvió de caldo de cultivo para el acelerado desarrollo de otra actividad esencialmente especulativa: la brutal "industria" del narcotráfico, cuyas exorbitantes ganancias no residen tanto en el "valor agregado" como en la enorme diferencia de precios entre América Latina y los Estados Unidos.

\section{La necesidad de la Ciencia y la Tecnología}

El hecho de que los países desarrollados sean a la vez los más industrializados y los que más esfuerzos dedican a la ciencia y la tecnología es algo más que una coincidencia. Desde mediados del siglo XVIII esos países ingresaron paulatinamente en la senda de construir unas economías basadas en la sustitución de la fuerza humana por las máquinas. Esto, a su vez, demandó la sustitución de la experiencia basada en la rutina por una aplicación consciente de las ciencias naturales.

Así por ejemplo, el primer motor de vapor fue posible gracias al gran desarrollo técnico alcanzado por el artesano inglés. Pero el mejoramiento de la máquina de vapor y el desarrollo de otros motores mejores y más eficientes ya no fue posible sin el desarrollo de la termodinámica, la electricidad, etc. es decir, de las ciencias naturales. Estas devinieron en una parte constitutiva indispensable, del aparato productivo de los países industrializados.

Esta característica, de la necesidad de la aplicación consciente de las ciencias naturales, se ha acentuado en las condiciones contemporáneas que comenzaron a conformarse a finales de la década de los treintas. Las máquinas e instrumentos 
construidos hasta estos años eran extensiones del músculo y de las capacidades sensoriales de los hombres. Pero los trabajos de N. Wiener (Cibernética), J. Von Neumann (teoría de juegos y cálculo binario) y $\mathrm{H}$. Aiken (primer ordenador, el Mark I) dieron inicio a un nuevo tipo de máquina basado en el control automático, el procesamiento de información y la retroalimentación, que representa una extensión de la capacidad humana de toma de decisiones. Con el desarrollo del transistor y los circuitos impresos, la conformación de equipos interdisciplinarios de expertos (Centros de Investigación y Desarrollo) y el perfeccionamiento de las máquinas de este nuevo tipo; surgen en los años setentas la moderna tecnología, ya no como «la ciencia o el estudio de las artes prácticas o industriales» sino como el estudio sistematizado del diseño, construcción y aplicación de máquinas y procesos basados en el principio de retro-alimentación a las actividades de los hombres.

Los últimos veinte años han sido testigos de grandes progresos de la tecnología, algunas de cuyas tendencias actuales, discriminadas por áreas claves del desarrollo industrial, son las siguientes:

- Generación y aprovechamiento de Energía.

La tendencia ha sido hacia la reducción en las necesidades de potencia de las máquinas. Esto ha posibilitado que nuevas formas de utilización de energía, como las celdas solares, encuentren cada vez más aplicaciones. Los grandes proyectos hidroeléctricos y termoeléctricos podrían devenir obsoletos a la vuelta de unas pocas décadas.

\section{- Procesamiento de Materiales}

La tendencia hacia la miniaturización, las capas delgadas y el creciente uso de nuevas cerámicas, nuevos compuestos y los productos de la biotecnología; hace prever que la disminución en la importancia de ciertos recursos naturales (cobre, hierro, níquel, etc.) y por ende de sus precios en los mercados internacionales sea un fenómeno persistente.

- Producción de Máquinas e Instrumentos

La microelectrónica afectará en el mediano plazo al $50 \%$ de las actividades productivas y un porcentaje similar de los puestos de trabajo, enfatizando la tendencia hacia máquinas y procedimientos pequeños y flexibles controlados por computadores.

El final de las grandes instalaciones industriales y los procesos de fabricación en serie, así como de la ventaja comparativa de poseer mano de obra barata, parece estar cercano

En conjunto todas las tendencias de la tecnología contemporánea apuntan hacia el desplazamiento de la mano de obra básica por una fuerza laboral con amplios conocimientos científicos y tecnológicos; hacia la reducción de las ventajas comparativas de clima, recursos naturales y mano de obra barata sobre las cuales han depositado sus esperanzas países subdesarrollados; y , hacia el aumento de la importancia de una mayor productividad y de un puesto de avanzada en el manejo y producción de ciencia y tecnología.

Las políticas de industrialización en Colombia han estado basadas en la sustitución de importaciones, a compra de tecnología y la desigual política de asociación mencionada arriba. Además la dependencia de la industria colombiana del sector externo no ha estado 
limitada al lado de la oferta (adquisición de máquinas, materias primas procesadas y tecnología extranjeros, sino también a lo relacionado

con la demanda debido a los continuos esfuerzos oficiales para aumentar las llamadas "exportaciones menores" ("exportar para progresar"). Sobra decir que no ha habido nunca una decisión de industrializar al país sobre la base de la generación interna de ciencia y tecnología, a pesar de 20 años de debates nacionales sobre la importancia de éstas para el progreso nacional y de la existencia de Planeación Nacional, de Colciencias y de un aparato universitario que se actualizó a finales de los sesentas.

En resumen, no se han transcendido los límites impuestos por la división internacional del trabajo, lo cual ha traído como consecuencia los bien modestos indicadores reseñados atrás. Lo que queda claro es que Colombia jamás podrá aspirar a otra cosa que a un lento crecimiento económico y a unas costosas modernizaciones periódicas mientras no transcienda esos límites, es decir, mientras no haya una intención de desarrollo nacional autónomo. Por otro lado, la íntima vinculación entre industrialización, desarrollo económico y desarrollo científico tecnológico; y, las tendencias contemporáneas de reducción de las ventajas comparativas como efecto de los desarrollos tecnológicos, también dejan muy en claro que sin una política de aclimatación, adaptación y generación interna de ciencia y tecnología, esa intención de desarrollo nacional autónomo no podrá convertirse en realidad.

\section{UNA POLITICA DE DESARROLLO NACIONAL AUTONOMO TIENE QUE PARTIR DE PONER EL ENFASIS EN EL DESARROLLO DEL MERCADO INTERNO Y EN EL FORTALECIMIENTO DE LA CAPACIDAD NACIONAL DE GENEBACION DE TECNOLOGIA Y CIENCIA.}

\subsection{La importancia del Mercado Interno}

Como lo señalamos arriba, uno de los axiomas de la planeación económica por el estado colombiano ha sido la imposibilidad del desarrollo nacional sin los capitales extranjeros. Se ha complementado este axioma con los argumentos de que la competitividad exige unas escalas de producción que superan en mucho el tamaño del mercado nacional y que la protección arancelaria incuba la ineficiencia. Se adoba lo anterior con disertaciones acerca de las estrechas vinculaciones que han conformado un sistema económico mundial único, y con el imperativo de que Colombia tenga una economía abierta al mercado internacional de capitales y manufacturas.

La historia pasada y reciente de los países industrializados, sin embargo, apunta en otra dirección. Los Estados Unidos, por ejemplo, lograron su desarrollo industrial básicamente aislados del mercado internacional, aislamiento que sólo se rompe en 1917 con su participación en la Primera Guerra Mundial. Esto también es cierto de Alemania y Japón. En este último país, a guisa de otro ejemplo, las empresas con mayor éxito en los mercados internacionales son aquellos que primero capturaron una porción importante de su mercado nacional, en una competencia intestina que raya en la ferocidad.

Además, incluso aquellos países industrializados que posan de paladines de la libertad de empresa y comercio han acudido sin sonrojo alguno a medidas proteccionistas cuando así lo demandan sus propias necesidades internas.

Por otra parte, el tamaño de un mercado interno no depende del número absoluto de habitantes de un país, sino de su capacidad adquisitiva y la ampliación del consumo 
interno no depende tanto del consumo personal como del consumo productivo. El proceso de industrialización, como lo demuestra la historia de los países desarrollados desde el siglo XVIII, es un ciclo de crecimiento de la producción industrial en el cual unas industrias sirven de mercado a otras; la parte más dinámica corresponde a la producción de materiales, máquinas y energía, es decir, al consumo para la producción. A su vez el consumo personal de la población aumenta por cuanto se especializa su tarea productiva como trabajadores de fábrica.

Ha sido precisamente porque el ciclo nunca se cerró en Colombia (nunca se consolidó el mercado interno), que el país no ha podido desarrollarse en este siglo básicamente debido a que el sector externo abastece las necesidades del consumo productivo de nuestra industria.

\subsection{La Capacidad Científico-Tecnológica Nacional}

Colombia es un país de ciclos vitales desarticulados. Además de esa desconexión en el ciclo de industrialización, y como producto de ella, nuestro sector productivo está desarticulado de la investigación y desarrollo en ciencia y tecnología; ésta aparece desvinculada de la Universidad; a su vez, la educación superior incide escasamente sobre los otros niveles de la educación y todo el sector educativo está lejos del sector productivo.

Como consecuencia, aunque formalmente tenemos industria, institutos y centros tecnológicos, comunidad científica, universidades que investigan, un fondo de fomento científico y tecnológico, y un sector educativo de amplia cobertura; cada uno de ellos languidece a la espera de la influencia externa.

Es evidente que esta múltiple desarticulación entorpece cualquier esfuerzo de desarrollo nacional autónomo, y que para superarla es necesaria una decisión desde el poder del Estado. La discusión hasta aquí ha pretendido señalar que el nudo gordiano se encuentra en la desarticulación entre industria, ciencia y tecnología. El problema esencial a resolver para nuestro desarrollo autónomo es la elevación de la productividad de las labores urbanas y rurales, y ello no es posible sin la aplicación de la ciencia y la tecnología contemporáneas. Puesto que el problema no se puede atacar en todas las direcciones a la vez, se hace necesario definir sectores estratégicos en cada una de las áreas claves mencionadas atrás (generación y aprovechamiento de energía, procesamiento de materiales y producción de máquinas) junto con el abastecimiento de alimentación y vestuario para la población; para hacer esfuerzos a fin de asimilar lo más avanzado de la ciencia y la tecnología a nivel mundial en esos sectores, y ponerlo al servicio de la nación Colombiana.

Más que grandes inversiones en adquisición de equipos novedosos o programas de becas en el exterior, se precisa racionalizar la utilización de los actuales recursos humanos, técnicos y financieros, articulándolos en una política de desarrollo. Un ejemplo lo constituye el SENA : Una Institución con un importante presupuesto propio, con la dotación en equipos e instrumentos sin paralelo en el país, con unos 10.000 profesionales y técnicos altamente calificados en casi todas las actividades económicas, con estrechos nexos con el sector empresarial y con convenios de cooperación técnica nacionales e internacionales; ampliamente subutilizada atendiendo solo unos 8.000 aprendices y 25.000 técnicos en capacitación, apoyando las improductivas actividades microempresariales. Esta entidad, con sus activos actuales podría convertirse en un 
centro para la investigación y desarrollo tecnológico del país. Nuevamente, se trata de una reorientación de recursos existentes.

\subsection{Una Prioridad: La Investigación y Desarrollo Tecnológicos}

Como anota un estudioso del tema (7),la principal diferencia entre la investigación científica y la tecnológica estriba en que mientras los autores de la primera se esfuerzan por divulgar sus hallazgos, la segunda se trata de mantener lo más secreta posible tras grandes barreras de patentes, licencias y otros medios de sigilo industrial. Esta diferencia ilustra bien la distinción entre la naturaleza de la ciencia y de la tecnología, a pesar de su íntima relación.

La ciencia busca la explicación de los cambiantes patrones de comportamiento de la naturaleza y la sociedad humana, mientras que la tecnología enfatiza en la manipulación de esos patrones de regularidad para el uso del hombre, así la explicación no esté siempre suficientemente clara. Fungiendo como una intersección entre ciencia e industria, la tecnología permite producir métodos y procesos retroalimentados para conseguir resultados definidos y minimizar el riesgo de fracasos en diferentes circunstancias.

Lo anterior tiene una implicación importante para una política de desarrollo nacional autónomo. Sin desdeñar la necesidad de conocimientos e investigación científica; nuestro país deberá, en primera instancia, enfatizar los esfuerzos en investigación y desarrollo tecnológicos. Las razones son bien claras: En primer lugar, es en la investigación y desarrollo tecnológicos que la ciencia encuentra su aplicación directa en la producción, con sus implicaciones sobre la elevación de la productividad y el progreso nacionales. En segundo lugar, la transferencia de tecnología de otros países, especialmente de los Estados Unidos, toma la forma de licencias para la utilización, no la apropiación, de los modernos desarrollos tecnológicos; esto presenta un doble obstáculo para el progreso nacional. Por un lado, los pagos en regalías representan un desangre de la riqueza nacional adicional a la deuda externa por unas tecnologías que normalmente son obsoletas o están cercanas a su obsolescencia en sus países de origen. Por el otro, representan una fuerte competencia contra los débiles esfuerzos de nuestros industriales. En palabras del periodista argentino, Luis Alonso, quien calculó para su país un giro anual de US\$ 500 millones por este concepto: “... los países de la región (Latinoamericana).., tienen la urgencia de incorporar intensivamente tecnología para reconvertir sus sistemas productivos, no sólo por cuestiones de orden económico, sino por la propia supervivencia de sus nacionalidades. De no ser así, ante el actual impulso de la transnacionalización que ya alcanzó el $40 \%$ de la economía mundial, se asistirá a una modernización impuesta, a una mayor dependencia".

\section{NOTAS}

1. Ver por ejemplo los artículos del autor, "La Tecnología Contemporánea y sus implicaciones en la Educación”, Educación y Cultura. Revista CEID-FECODE, No. 17. Bogotá, Junio de 1989; "La Educación en Ciencia y Tecnología y los Problemas del Desarrollo Nacional". Didaskalia. Cuadernos Docentes. Asociación de Profesores UPN, No. 3, Bogotá, Nov. 1989.

2. Nelson Rockefeller, "La Calidad de la Vida en las Américas". Informe al Presidente de los Estados Unidos de América. Washington, D.C., 1969. Lo referente al caso del 
CAME puede verse por ejemplo en "Las Relaciones Económicas entre Cuba y la Unión Soviética". Novedades. Agencia de Prensa NOVOSTI. Moscú, Octubre, 1974.

3. Citados por Francisco Restrepo. "El Mundo en el año 2.000". Conferencia ante el VII Congreso Nacional de Tecnólogos. Medellín, Marzo, 1989.

4. Entre los numerosos estudios dedicados al tema merecen citarse: Forero R. y otros (Compiladores y ed.) "Documentos sobre el Cerrejón". Ancora Editores, Bogotá, 1985; Acosta, A. "Cerromatoso S.A. Itinerario de un Zarpazo". Fondo de Publicaciones de la Cámara de Representantes. Riohacha, 1986.

5. Ver entre otros, Ominami C.; Pérez C.; Boyer R., "La Tercera Revolución Industrial". Ed. GEL. Buenos Aires, 1986.

6. Ver por ejemplo la discusión en varios aut. "Hacia una Nueva Colombia Industrial". Economía Colombiana. Revista de la Contraloría General de la República. Nos. 187188.Bogotá, Nov. - Dic., 1986.

7. Ben - David, Joseph. "El Empresariado Científico y la utilización de la Investigación», en estudios sobre la Sociología de la Ciencia, Alianza Universal, Madrid, 1980. 